\title{
Quatre hypothèses comparatives France-Pologne sur la violence antisémite au XXe siècle (Partie 1)
}

\section{Paul Zawadzki}

\section{(2) OpenEdition}

\section{Journals}

Édition électronique

URL : http://journals.openedition.org/conflits/413

DOI : $10.4000 /$ conflits.413

ISSN : $1777-5345$

Éditeur :

CCLS - Centre d'études sur les conflits lilberté et sécurité, L'Harmattan

Édition imprimée

Date de publication : 15 mai 1993

ISSN : 1157-996X

\section{Référence électronique}

Paul Zawadzki, «Quatre hypothèses comparatives France-Pologne sur la violence antisémite au XXe siècle (Partie 1) », Cultures \& Conflits [En ligne], 09-10 | printemps-été 1993, mis en ligne le 27 janvier 2003, consulté le 30 mars 2021. URL : http://journals.openedition.org/conflits/413 ; DOI : https:// doi.org/10.4000/conflits. 413

Ce document a été généré automatiquement le 30 mars 2021 


\title{
Quatre hypothèses comparatives France-Pologne sur la violence antisémite au XXe siècle (Partie 1)
}

\author{
Paul Zawadzki
}

1 Les recherches d'inspiration anthropologique, psycho-sociologique ou psychanalytique, qui se sont multipliées depuis la découverte du génocide ont souvent tenté d'expliquer l'antisémitisme, par le biais de théories à vocation universelle, en privilégiant son caractère diachronique et largement européen. Tout en essayant de faire la part de l'universel et du spécifique, la perspective ouverte par la sociologie historique comparative met au contraire l'accent sur les différentes formes de l'antisémitisme. Car, vu de plus près, les modes d'expression et manifestations concrètes de l'antisémitisme, sa capacité de mobilisation sont extrêmement différenciées en fonction des contextes socio-historiques.

2 Ainsi s'agissant de la violence antisémite, comment ne pas être frappé par le contraste France/Pologne. A partir de la fin du XIXe siècle, on observe une expansion de la violence antisémite physique, collective et individuelle, en Pologne, alors qu'en France ${ }^{1}$, au contraire, cette violence (physique) se marginalise. Certes, il y eut encore entre janvier 1898 et août 1899 des émeutes antijuives dans un grand nombre de villes en France $^{2}$. Mais en dépit de la montée de l'antisémitisme dans les années trente, celui-ci ne se manifeste pas sous la forme de pogromes. Ce n'est pourtant pas faute de violence. Pierre Birnbaum a récemment rappelé l'extraordinaire haine véhiculée par toute une floraison de textes, pamphlets et libelles antisémites en tous genres ${ }^{3}$. La France juive de Drumont (1886) connût 200 éditions jusqu'en $1914^{4}$. De telle sorte qu'on peut se demander "pourquoi n'y a-t-il pas eu de pogromes en France au XXe siècle ?".

3 Le cas français montre d'ailleurs la complexité de la question du passage à l'acte. Sans même rappeler les difficultés liées au concept d'explication en sciences sociales, la multiplication des facteurs explicatifs ou la construction de modèles n'élucide pas l'énigme du passage à l'acte. En se demandant "pourquoi n'y a-t-il pas de socialisme aux Etats-Unis ?", Sombart esquissait un modèle reposant sur l'action stratégique de 
l'individu rationnel. Il est certainement possible de rendre compte des facilitations de la violence antisémite, tant par la structure de la situation dans laquelle elle se produit, que par les croyances des acteurs, ou leur "rationalité subjective" 5 (par exemple la légende du meurtre rituel), mais il est difficile de réduire les pogromes à la seule violence stratégique. De plus, comme le racisme, l'antisémitisme peut s'exprimer sous forme de préjugé ou d'idéologie, ou bien s'incarner dans des pratiques de discrimination, allant de la ségrégation jusqu'au meurtre ${ }^{6}$. Or comme le montre, par exemple, la célèbre expérience de LaPiere ${ }^{7}$, il n'y a pas de relation de nécessité entre préjugé et pratique. De même, on est loin du consensus dans la résolution du problème historique et épistémologique du passage de la doctrine au meurtre et, comme l'écrit de manière provocante Jacob Katz, "attribuer les effets mortels de l'antisémitisme à la pensée raciste, c'est comme imputer les effets mortels des couteaux à leur inventeur"8. Reste qu'un pogrome sans préjugés antisémites est improbable et que d'une manière générale, si l'on en croit Ted Gurr, le potentiel de la violence politique est corrélé avec les justifications normatives et instrumentales qu'on lui confère 9 .

4 L'antisémitisme en Pologne au XXe siècle ne fut pas seulement préjugé, idéologie, ou instrument de mobilisation. Il prit également la forme d'une pratique sanglante, bien que le singulier soit ici trompeur: le déferlement de la violence antisémite (physique) entre 1918 et 1947 n'obéit probablement pas à une logique unique. Ses enjeux et ses détonateurs sont variables suivant les situations. Reste que son ampleur est sans précédent dans l'histoire de la Pologne puisque celle ci n'a jamais connu les massacres médiévaux qui, en Occident, se chiffrent par milliers de morts entre la première croisade (XIe) et la grande peste (XIVe). En voici les manifestations les plus spectaculaires, que l'on est d'autant plus contraint de rappeler qu'elles furent longtemps l'objet d'une dénégation de l'historiographie polonaise.

5 L'indépendance s'ouvre sur une première vague de pogromes (Cracovie, Kielce, Lublin, Przemysl, etc.) ${ }^{10}$ et dont le plus connu est peut être celui de Lwow le 22 novembre 1918 (au moins une centaine de morts) ${ }^{11}$. On note une seconde vague de violence antisémite dans les années 1929-1931, mais celle ci s'exacerbe principalement dans la seconde moitié des années trente. Sans viser l'exhaustivité, citons les pogromes de Grodno, Suwalki (juin 1935), Odrzywol (novembre 1935), Czyzew (décembre 1935), Przytyk (mars 1936), Minsk Mazowiecki (juin 1936), Brzesc (mai 1937), Czestochowa (juin 1937), Bielsko Biala (septembre 1937) etc ${ }^{12}$. Des rapports internes du Ministère de l'Intérieur indiquent une échelle quantitative de la violence. Pour le troisième trimestre 1936, on note 92 agressions individuelles et 105 agressions collectives, 571 blessés et 26 tués, 1115 cas de vitres brisées ${ }^{13}$. Au quatrième trimestre, les mêmes sources indiquent 654 blessés, 13 tués, 1492 cas de vitres brisées ${ }^{14}$. Entre 1935 et 1937, on a pu dénombrer une cinquantaine d'attaques à la bombe visant généralement des magasins juifs, parfois des synagogues ${ }^{15}$.

6 Tout au long des années trente, des bagarres, déclenchées par les corporations nationalistes et fascisantes qui dominaient alors la vie politique étudiante, ensanglantent les Universités et les écoles supérieures ${ }^{16}$. Loin de mettre un coup d'arrêt aux violences populaires, les débuts de l'occupation semblent au contraire les exacerber. En consignant jour après jour, avec la passion de l'ethnologue qui voit sombrer sa propre société, la prolifération des violences antijuives à Varsovie, entre octobre 1939 et novembre 1940, l'historien Emanuel Ringelblum observait que "dans certaines rues le Juif ne pouvait se montrer, de peur de rentrer chez lui, battu jusqu'au 
sang, dépouillé de son argent et même de ses vêtements. La rue devint le lien entre les antisémites polonais et les hitlériens"17. Ni le génocide ni la fin de la guerre ne mettent un terme à la violence antisémite. Selon les chiffres disponibles, 150 Juifs furent assassinés au cours du premier trimestre $1945^{18}$, 353 sur l'ensemble de l'année ${ }^{19}, 800$ jusqu'en avril $1946^{20}$. Jusqu'en août 1946, les "actions ferroviaires" consistant à piller les Juifs (ou supposés) dans les trains, puis à les assassiner firent plus de 120 victimes $^{21}$. L'auteur du Massacre des survivants estime que 1500 Juifs furent tués dans ces années d'après guerre ${ }^{22}$. En juin 1945 des émeutes antijuives se produisent à Rzeszow. Des pogromes éclatent le 11 août 1945 à Cracovie (2 morts), le 4 juillet 1946 à Kielce (42 morts) ${ }^{23}$. La Pologne n'est pas le seul pays d'Europe de l'Est où des pogromes eurent lieu après la guerre. Qu'il suffise de rappeler les violences de Kunmadaras en Hongrie (mai 1946) ${ }^{24}$, celles de Velke Topolcany en Slovaquie (septembre 1945) ${ }^{25}$. Mais c'est assurément celui où la violence antisémite diffuse sur l'ensemble du territoire eut le plus d'ampleur, là où elle fit le plus de victimes. Car, hormis les pogromes, on est frappé, dans les campagnes, par la fréquence, des assassinats ponctuels des survivants revenant sur leurs anciens lieux d'habitation, récupérer parfois des biens qui leur avaient appartenu. Poursuivant, en 1984, ses recherches sur l'image du Juif dans la culture populaire (rurale), l'ethnologue Alina Cala note que, partout où la question des incidents violents fut posée, les enquêtés se souvenaient de meurtres commis sur des individus isolés, des groupes ou des familles juives ${ }^{26}$.

Dans quelle mesure la sociologie historique du politique peut-elle éclairer le contraste France/Pologne? Loin d'être exhaustives, les quatre hypothèses retenues ici sont liées aux trajectoires inverses des constructions politiques respectives. Elles "n'expliquent" pas la violence antisémite mais contribuent à rendre compte de certaines de ses facilitations à l'Est, et en ce sens elles privilégient les spécificités du cas polonais. C'est pourquoi on abordera pas ici ce qui relève du "patrimoine" européen des préjugés antisémites. Par exemple seront passés sous silence les avatars modernes de la diabolisation médiévale du Juif qui resurgit si fréquemment dans le discours antijuif "d'auto-victimisation"27, et dont le mythe du complot juif mondial mis en forme au XXe par les Protocoles des Sages de Sion constitue le paradigme. Dans les exemples choisis nous avons privilégié la flambée de violence antisémite des années trente en Pologne, car non seulement son initiative est très souvent politique (groupes nationalistes et fascisants), mais surtout parce que, conformément aux critères définitionnels de la violence politique retenus dans le projet même de cette table ronde, elle "influence les rapports de forces socialement constitués ainsi que les modes de gouvernement qui tentent de les régir" ${ }^{\prime 2}$. De plus, cette violence a des effets sur les stratégies politiques des Juifs polonais qui s'orientent davantage qu'en France vers la protestation (communisme, socialismes) ou la sortie (sionisme). L'expérience des années trente est fondamentale en Pologne en tant qu'elle constitue une rupture du contrat hobbesien qu'on peut traduire par "le lien stratégique entre l'abandon par l'individu-citoyen de sa liberté politique et l'accomplissement par l'Etat d'une fonction de protection"29, ce qui à son tour permet de comprendre pourquoi les stratégies politiques des Juifs furent dans l'ensemble plus radicales en Pologne qu'en France.

Violence et centralisation

8 La première hypothèse reste sans doute la plus fragile et attend encore son historien. $\mathrm{Si}$ l'on accepte dans la perspective de Norbert Elias, qui "peut n'apparaître que comme un essayiste de talent" ${ }^{130}$, l'hypothèse longue d'une pacification des conduites en Occident, liée notamment au développement de l'Etat (avec pour la France le processus de 
"curialisation des guerriers"), hypothèse qui, dans sa généralité, semble confirmée par certains historiens ${ }^{31}$, le contraste polonais apparaît immédiatement. En effet ce qui constitue la spécificité historique fondamentale de la Pologne à partir de la fin du XVIe siècle c'est la "décentralisation de la souveraineté" ${ }^{32}$. Le succès du refus nobiliaire de la délégation de la puissance au profit du centre politique marque "la victoire des périphéries"33. Dans une Europe où s'affirment les absolutismes, l'échec de la centralisation politique signifie l'impossibilité pour l'Etat de se constituer en monopole de la coercition physique légitime ${ }^{34}$ et d'étendre son pouvoir de tutelle sur l'ensemble du territoire, tant sur le plan de la justice que celui de la police. La fameuse "liberté dorée" des nobles c'était d'un côté le "jus vitae et necis" (codifié en 1574) qui établissait le droit de vie et de mort sur les serfs, et de l'autre "l'habitude de mener de front l'action judiciaire légale et d'agir simultanément par la force brutale", ce qu'on appelait "faire valoir ses droits jure et gladio"35. L'oligarchie nobiliaire se rendait justice elle même ${ }^{36}$. Comme le résume de manière plaisante un des initiateurs polonais de l'anthropologie historique, commentant ainsi la pratique des incursions nobiliaires ${ }^{37 "}$ : "Le noble estimait que Dieu est trop haut et le Roi trop loin pour qu'ils puissent rendre justice rapidement, aussi il la rendait lui même; il s'emparait militairement de la maison voisine, prenait le voisin et sa famille en otage et l'obligeait à accepter les conditions de l'accord" ${ }^{\prime 38}$. D'une manière générale, entre le XVIe et le XVIIIe siècle, les occasions de prendre les armes étaient très nombreuses : sans même évoquer les luttes internes entre magnats, par petite noblesse interposée, "des réunions de dizaines ou de centaines d'individus armés, lors d'une noce ou d'une fête de baptême, ou encore dans une diétine, suffisaient à constituer une masse critique : pour la moindre raison on saisissait le sabre, ou parfois simplement des haches, des bâtons ou des ranches" 39 . C'est dire qu'avec la décentralisation et la persistance de ces "guerres du dedans" ${ }^{40}$, on est loin du modèle de domestication des pulsions et des affects lié à la monopolisation de la contrainte que décrit Elias ${ }^{41}$.

Devenue périphérie d'Empires, "la Pologne a-t-elle perdu le XIXe siècle sous l'angle de la civilisation moderne?" En dépit de la connotation évolutionniste d'une telle question, les historiens en débattent périodiquement ${ }^{42}$. La socialisation politique des masses, l'apprentissage de la démocratie permettant l'intégration de la protestation dans un jeu politique institutionnalisé devront attendre le XXe siècle. Tout au long du XIXe siècle le recours à la violence reste fréquent. Glorifiées par le romantisme, les insurrections contre un ordre politique dépourvu de légitimité culturelle étaient réprimées dans le sang, tout comme le furent les protestations ouvrières, souvent proches de l'émeute (par exemple Lodz 1892), au tournant du siècle. Par ailleurs, les normes coutumières perpétuaient l'injonction " $d$ 'avoir le courage de tuer un homme lorsque celui-ci porte atteinte à l'honneur"43. La noblesse polonaise dans l'empire russe sera politiquement décapitée mais l'image des campagnes polonaises, où sévissent au XIXe et au début du XXe siècle alcoolisme et criminalité ${ }^{44}$, indique une tendance au maintien d'une violence rurale qui paraît parfois d'une banalité étonnante. "Jamais une noce ne pouvait se dérouler sans bagarre", raconte un violoniste de la fin du XIXe siècle ${ }^{45}$. Dans ses mémoires, le grand leader du parti paysan P.S.L., W. Witos (1874-1945), rapporte que "les bagarres entre valets de ferme des différents villages étaient à l'ordre du jour. Celles-ci étaient inévitables dans les fêtes, les noces, et surtout à l'auberge. Elles mettaient aux prises deux individus, (...) ou bien revêtaient un caractère de masse, lorsqu'à la bagarre se joignaient les amis des parties en présence. Très souvent certains villages faisaient de véritables expéditions guerrières contre les villages voisins. Une 
bataille perdue ou gagnée fournissait toujours la raison d'une nouvelle guerre, qui durait des années"46.

Si la proposition probable, mais difficile à contrôler, d'une persistance historique de la violence plus importante en Pologne qu'en France est vraie, il est clair que la violence antisémite au XXe siècle devrait d'abord être replacée dans ce cadre général. Ajoutons que la majeure partie du territoire polonais, là où les Juifs étaient les plus nombreux, fut incorporée à l'Empire russe qui inventa "une nouvelle technique antisémite"47 faisant des pogromes un mode de gouvernement. Le cas des pogromes organisés par les autorités russes (Varsovie 1881, Bialystok et Siedlce 1906) dépasse les limites de cet article mais la question demeure : comment évaluer à long terme les conséquences culturelles de cette autorisation officielle de tuer des Juifs? Que l'on accepte ou non l'idée d'une persistance historique de la violence plus importante en Pologne qu'en France, il reste que le monopole de la coercition physique par l'Etat, ainsi que son emprise sur la société et sur l'ensemble du territoire sont, très directement cette fois, en question dans les pogromes du XXe siècle. Ceux ci se produisent dans des moments, où la puissance de l'Etat est vacillante. Ce fut le cas au lendemain des deux guerres mondiales. La vague de violences entre 1935 et 1937 se produit après la mort de Pilsudski, figure tutélaire de l'autoritarisme polonais, pendant la "décomposition" du camp gouvernemental et la crise de légitimité du pouvoir qui s'en suivirent. De même, les pogromes au lendemain de la seconde guerre mondiale se sont déroulés dans le cadre du processus de la prise de pouvoir communiste, dans des conditions proches de la guerre civile. La récente publication de rapports officiels concernant le pogrome de Kielce (juillet 1946), n'accréditent pas davantage la thèse de la provocation soviétique ${ }^{48}$ (jamais démontrée) mais révèlent en revanche un climat d'antisémitisme parmi la base du Parti, dans l'armée et dans la milice ${ }^{49}$. Des recherches récentes attestent que le rôle de la milice et de l'armée pendant le pogrome de Kielce ne s'est pas limité à la passivité : certains fonctionnaires ont épousé la cause des pogromistes ${ }^{50}$.

11 Il semble enfin que la violence antisémite au cours de l'entre-deux-guerres se soit davantage exprimée aux franges des confins orientaux, ces territoires historiquement agités (massacres de Chmielnicki au XVIIe siècle), partagés entre Polonais, Ukrainiens, Biélorusses, Lituaniens et Juifs, que l'Etat polonais s'efforçait de "coloniser" et de "poloniser" au cours de l'entre-deux-guerres. Lorsqu'ils nous fournissent des statistiques, les rapports officiels détaillent parfois les chiffres des régions de l'Est. Dans la seconde moitié de 1936, sur 2607 cas de vitres brisées répertoriées, 1660 se produisirent dans la voïvodie de Bialystok ${ }^{51}$, à la frontière Est de l'actuelle Pologne.

Lire la suite

\section{NOTES}

1. Notre propos ne concerne que la métropole car il y eut des pogromes en Algérie (à Constantine en 1934 notamment). 
2. Wilson (S.), Ideology and experience. Antisemitism in France at the Time of the the Dreyfus Affair, London and Toronto, Associated University Press, 1982, en particulier le chapitre "The antisemitic riots of 1898", pp. 106-124; Michael R. Marrus, Les Juifs de France à l'époque de l'affaire Dreyfus. L'assimilation à l'épreuve (trad.), Paris, CalmannLévy, 1972, pp. 240-243 ; Zeev Sternhell, La droite révolutionnaire. Les origines françaises du fascisme 1885-1914, Paris, Seuil/Points, pp. 230-239.

3. Un mythe politique : la "République juive", de Léon Blum à Pierre Mendès France, Paris, Fayard, 1988.

4. Michel Winock, Nationalisme, antisémitisme et fascisme en France, Paris, Seuil/ Points, 1990, p. 118.

5. Raymond Boudon, L'art de se persuader des idées fausses, fragiles ou douteuses, Paris, Fayard, 1990, pp. 373 et suiv.

6. Ce sont les trois niveaux d'interrogations dégagées par Michael Banton, Sociologie des relations raciales (trad. 1ere éd. anglaise, 1967), Paris, Payot, 1971, p. 18.

7. En traversant les Etats Unis dans les années trente avec un couple d'amis chinois, LaPiere s'arrêta dans 67 hôtels dont un seul refusa de les loger et 84 restaurants qui les reçurent tous. Six mois après son retour, il leur adressa un questionnaire. A la question "Accepteriez vous des individus de race chinoise comme clients dans votre établissement ?", plus de $90 \%$ des propriétaires d'hôtels et de restaurants (qui repondirent) ont déclaré qu'ils n'accepteraient pas de clients chinois, cf. Richard T. Lapiere, "Attitudes versus Actions", Social Forces $\mathrm{N}^{\circ} 13,1934$, cité par Michael Billig, "Racisme, préjugé et discrimination", in Serge Moscovici (éd.), Psychologie sociale, Paris, P.U.F, 1984, pp. 451-452.

8. Jacob Katz, "Lectures défectueuses de l'antisémitisme"(trad.), Sens, N5-6, 1984, p. 208.

9. Ted Robert Gurr, Why Men Rebel (1970), Princeton, Princeton University Press, 1974, p. 156.

10. Israel Cohen, A Report on the Pogroms in Poland, Central Office of the Zionist Organisation, april 1919, (repris in Seeds of Conflict Series 2, Palestine, Zionism and the Levant 1912-1946, Tome 1, The Roots, Nendeln, Klaus Reprint, 1974) qui énumère près de 130 localités touchées par des violences antijuives entre décembre 1918 et janvier 1919 (pp. 11-20) ; Les Pogromes Anti-Juifs en Pologne et en Galicie en novembre et décembre 1918. Faits et Documents réunis et rédigés par L. Chasanowitch, Stockholm, 1919.

11. Jerzy Tomaszewski, "Lwow, 22 listopada 1918" (Lwow, 22 novembre 1918), Przeglad Historyczny $\mathrm{N}^{\circ} 2,1984$, pp. 279-285.

12. Tatiana Berenstein, "KPP $\mathrm{w}$ walce $\mathrm{z}$ pogromami antyzydowskimi $\mathrm{w}$ latach 1935-1937" (Le Parti Communiste Polonais dans la lutte contre les pogromes antijuifs dans les années 1935-1937), B. Z. I. H., N¹5-16, 1955, pp. 19-21 en particulier ; Artur Eisenbach, préface à Emanuel Ringelblum, Stosunki polsko-zydowskie w czasie drugiej wojny swiatowej (Les relations judéo-poloniases pendant la seconde guere mondiale, 1943), Warszawa, Czytelnik, 1988, pp. 8-9 ;

13. Sprawozdanie z zycia mniejszosci narodowych za III kwartal 1936, (Rapport concernant la vie des minorités nationales pour le III trimestre 1936), Ministerstwo Spraw Wewnetrznych, wydzial narodosciowy, Warszawa, 1936, p. 10.

14. Sprawozdanie z zycia mniejszosci narodowych za IV kwartal 1936 (Rapport concernant la vie des minorités nationales pour le IV trimestre 1936), Ministerstwo Spraw Wewnetrznych, wydzial narodowosciowy, Warszawa, 1936, p. 17. 
15. Jolanta Zyndul, "Zajscia antyzydowskie w Polsce w latach 1935-1937, geografia i formy" (Incidents antijuifs en Pologne dans les années 1935-1937, géographie et formes), B.Z.I.H., N³, 1991, p. 59.

16. Sur ce point, on dispose des témoignages de deux classiques de la sociologie polonaise : Ludwik Krzywicki,"Burdy studenckie" (bagarres étudiantes), in Wspomnienia (Mémoires), Warszawa, Czytelnik, 1959, tome III, pp. 285-384 ; Stefan Czarnowski, "Zajscia antysemickie w szkolach wyzszych (Incidents antisémites dans les écoles supérieures), Dziela (OEuvres), P.W.N., 1956, tome V, pp. 55-62 ; voir également Andrzej Pilch, Studencki ruch polityczny w Polsce w latach 1932-1939 (Le mouvement politique étudiant en Pologne 1932-1939), Krakow, Uniwersytet Jagiellonski, 1972, pp. 150-169.

17. Emanuel Ringelblum, Stosunki..., op. cit., pp. 48 et 50.

18. Chiffres du Comité Central Juif en Pologne, (C.K.Z.P.).

19. Article "Poland", Encyclopaedia Judaïca, p. 784.

20. Bernard D. Weinryb, "Poland", in Peter Meyer et al. The Jews in the Soviet Satellites, Syracuse, Syracuse University Press, 1953, p. 252.

21. Krzysztof Urbanski,"Z problematyki zydowskiej w kieleckim w latach 1945-1946" (De la problématique juive dans la région de Kielce 1945-1946), in Feliks Kiryk (éd.), Zydzi w Malopolsce (Les Juifs en Petite Pologne), Przemysl, Poludniowo-Wschodni Instytut Naukowy, 1991, p. 349.

22. Marc Hillel, Le massacre des survivants. En Pologne après l'holocauste (1945-1947), Paris, Plon, 1985, p. 351.

23. Sur le pogrome de Kielce, nous disposons maintenant de nombreux travaux : Stanislaw Ossowski, "Na tle wydarzen kieleckich", (1946), Kultura i spoleczenstwo, N¹, 1987, pp. 49-57 (l'article a disparu de ses oeuvres complètes publiées à Varsovie/P.W.N. entre 1966 et 1970) ; Krystyna Kersten, Polacy, Zydzi, Komunizm. Anatomia polprawd 1939-68 (Les Polonais, les Juifs, le communisme. Anatomie de demi vérités 1939-68), Warszawa, Niezalezna Oficyna Wydawnicza, 1992, pp. 89-142 ; Bozena Szaynok, Pogrom Zydow w Kielcach 4 VII 1946 (Le pogrome des Juifs à Kielce 4 VII 1946), Warszawa, Bellona, 1992.

24. Istvan Bibo, "La question juive en Hongrie après 1944", in Misère des petits Etats d'Europe de l'Est (trad.), Paris, l'Harmattan, 1986, p. 371 ; François Fejto, Les Juifs et l'antisémitisme dans les pays communistes (entre l'intégration et la sécession), Paris, Plon, 1960, p. 80.

25. Peter Meyer, "Czechoslovakia" in Peter Meyer et al., The Jews in the Soviet Satellites, op. cit., p. 105.

26. Alina Cala, "Wizerunek Zyda w polskiej kulturze ludowej" (L'image du Juif dans la culture populaire polonaise), in Polska mysl polityczna XIX i XX wieku (La pensée politique polonaise aux XIXe et XXe siècle), tome VIII, Wroclaw, Ossolineum, 1992, p. 221.

27. Pierre-André Taguieff, "Sur une argumentation antijuive de base : l'autovictimisation du narrateur", Sens, Nº, 1983, pp. 133-156.

28. Philippe Braud, "La violence politique dans les démocraties occidentales", 2 avril 1992, p.2.

29. Bertrand Badie, "Terrorisme et Etat", Etudes Polémologiques, N¹, 1989, p. 10. 30. Guy Hermet, "A propos de l'obstination historique", Revue Internationale des Sciences Sociales, $\mathrm{N}^{\circ} 133,1992$, p. 390. 
31. "C'est donc à l'émergence de la rationalité dans la sphère morale et à la soumission à la règle étatique qu'il faut imputer la marginalisation progressive de la violence dans les sociétés occidentales", cf. Jean-Claude Chesnais, Histoire de la violence en Occident de 1800 à nos jours, Paris, Pluriel, 1982 (éd. rev. et aug.), p. 16.

32. Juliusz Bardach, Boguslaw Lesnodorski, Michal Pietrzak, Historia Panstwa i Prawa polskiego, (Histoire de l'Etat et du Droit polonais), Warszawa, P.W.N., 5e éd. 1987, p. 225.

33. Antoni Maczak, Rzadzacy i rzadzeni. Wladza i spole-czenstwo w Europie wczesnonowozytnej (Gouvernants et gouvernés. Pouvoir et société en Europe moderne), Warszawa, P.I.W., 1986, p. 134 ; voir aussi Perry Anderson, l'Etat absolutiste, tome 2, l'Europe de l'Est, Paris, Maspero, 1978, pp. 99-120.

34. Au XVIIIe siècle l'Etat disposait d'une armée nationale de 16000 hommes, tandis que la noblesse en comptait 30000 et les voisins (Russie, autriche, Prusse ) entre 200 et 500 000, chiffres cités par Charles Tilly, Contrainte et capital dans la formation de l'Europe 990-1990 (trad. 1ere éd. 1990), Paris, Aubier, 1992, p. 235.

35. Alexandre Wolowski, La vie quotidienne en Pologne au XVIIe siècle, Paris, Hachette, 1972, p. 59.

36. Janusz Tazbir, Kultura szlachecka w Polsce, rozkwit, upadek-relikty (La culture nobiliaire en Pologne, épanouissement, chute, persistance), Warszawa, W.P., 3e éd. modifiée, 1983, p. 38-39.

37. zajazd

38. Jan Stanislaw Bystron, Dzeje obyczajow w dawnej Polsce, wiek XVI-XVIII (L'histoire des coutumes dans l'ancienne Pologne, XVI-XVIIIe siècles,1ère éd. 1932), tome 2, Warszawa, P.I.W., 3e éd. 1976, p. 318.

39. Ireneusz Ihnatowicz et al., Spoleczenstwo polskie od X do XX wieku (La société polonaise du Xe au XXe s.), Warszawa, K. i W., 2e éd. 1988 p. 402.

40. Georges Balandier, "La violence et la guerre : une anthropologie", Revue Internationale des Sciences Sociales $\mathrm{N}^{\circ} 110,1986$, p. 533.

41. Norbert Elias, La dynamique de l'Occident (trad. 1ere éd. 1939), Paris, CalmannLévy, 1975, pp. 187 et suiv.

42. Cf. Kwartalnik Historyczny, $\mathrm{N}^{\circ} 1,1979$, pp. 89-129.

43. Ireneusz Ihnatowicz et al., op. cit., p. 567.

44. Ludwik Stomma, Campagnes insolites. Paysannerie polonaise et mythes européens, Lagrasse, Verdier, 1986, p. 158-166.

45. cité par L. Stomma, idem, p. 162.

46. Wincenty Witos, Moje wspomnienia (Mes souvenirs), 1e partie, (1e éd. 1964), Warszawa, Ludowa Spoldzielnia Wydawnicza, 1988, pp. 113-114.

47. Léon Poliakov, préface à Simon Doubnov, Histoire d'un soldat juif 1880-1915, Paris, Cerf, 1988, p. 8 ; Léon Poliakov, Histoire de l'antisémitisme, Tome 4, l'Europe suicidaire 1870-1933, Paris, Calmann-Lévy, 1977, p. 102 et s/s ; John D. Klier and Shlomo Lambroza (ed.), Pogroms : Anti-Jewish Violence in Modern Russian History, Cambridge, Cambridge University Press, 1992.

48. Pour la thèse de la provocation, cf. Krystyna Kersten, "Kielce, 4 lipca 1946", Tygodnik Solidarnosc N³6, 1981 ; Michael Checinski, Poland. Communism, Nationalism, Anti-Semitism, New-York, Karz-Kohl Publishing, 1982, pp. 21-34.

49. Andrzej Paczkowski (ed.), "Raporty o pogromie" (Rapports concernant le pogrome), Puls, $N^{\circ} 3,1991$, pp. 101-121.

50. Bozena Szaynok, Pogrom Zydow w Kielcach, op. cit. 
51. Sprawozdanie z zycia mniejszosci narodowych za III kwartal 1936, op. cit., p. 10 ; Sprawozdanie z zycia mniejszosci narodowych za IV kwartal 1936, op. cit., p. 17.

\section{RÉSUMÉS}

A travers quatre hypothèses complémentaires liant la violence antisémite aux modes de construction du politique, l'auteur se propose de rendre compte de deux situations fortement contrastées au XXè siècle : expansion de la violence antisémite physique en Pologne ; marginalisation de cette violence en France. La première hypothèse reprend la thèse de Norbert Elias liant l'intériorisation de la violence à la centralisation politique, en rappelant le caractère inversé des modes de construction du politique en France et en Pologne. La seconde, inspirée de Tocqueville, tire les conséquences sur le plan de la violence antisémite du fait que l'égalisation des conditions fut longtemps bloquée à l'Est. La troisième porte sur la construction de la citoyenneté. En France, l'Etat parvient à imposer une citoyenneté universaliste, tandis que la faiblesse de l'Etat en Pologne, où l'idée républicaine n'a jamais pris racine, rend la situation des Juifs particulièrement précaire. La quatrième hypothèse jette un pont entre des travaux psychosociologiques et une analyse du politique. Tandis que dans un système à Etat fort, culture et politique sont fortement dissociés, en Pologne au contraire, la violence antisémite dans sa dimension passionnelle est grandement facilité par l'irruption des catégories identitaires et culturelles dans la sphère du politique et la dédifférenciation de l'Etat.

Using four complementary hypotheses, the author attempts to explain two radically different situations in the 20th century : the extension of physical anti-Semitic violence in Poland as opposed to its virtual disappearance in France. The first hypothesis is based on Norbert Elias' thesis linking the interiorisation of violence to political centralisation ; in this respect political development in Poland and in France appear radically opposed. The second hypothesis - drawn from Tocqueville - considers that anti-Semitic violence is due to delayed political equality in the East. The third relates to the development of the citizenship concept. In France, the State achieved the acceptance of universal citizenship, whereas in Poland the weakness of the State and the feeble roots of the republican ideal rendered the status of the Jews precarious. The fourth hypothesis links psycho-sociology to political analysis. Whereas in a strong State, culture and politics are clearly distinct, in Poland, emotional factors (of culture or identity) merge with politics and give to anti-Semitism an echo within the political sphere.

\section{INDEX}

Index géographique : Aires géographiques comparées, Europe centrale et orientale

Mots-clés : histoire, racisme, sociologie historique

Index chronologique : 20ème siècle 